\title{
Holographic entanglement entropy in insulator/superconductor transition with Born-Infeld electrodynamics
}

\author{
Weiping Yao and Jiliang Jing ${ }^{1}$ \\ Department of Physics, and Key Laboratory of Low Dimensional Quantum Structures \\ and Quantum Control of Ministry of Education, \\ Hunan Normal University, Changsha, Hunan 410081, P.R. China \\ E-mail: yao11a@126.com, jljing@hunnu.edu.cn
}

ABstRact: We investigate the holographic entanglement entropy in the insulator/superconductor phase transition for the Born-Infeld electrodynamics with full backreaction in five-dimensional AdS soliton spacetime. We note that the holographic entanglement entropy is a good probe to study the properties of the phase transition, and the Born-Infeld factor $b$ has no effect on the critical chemical potential $\mu_{c}$. We find that both in the half space and the belt one, the non-monotonic behavior of the entanglement entropy versus the chemical potential is a general property, and the entanglement entropy increases with the increase of the Born-Infeld factor in the superconductor phase. Particularly, there exists confinement/deconfinement phase transition in the strip geometry and the critical width $\ell_{c}$ is dependent of the Born-Infeld parameter.

KEYwords: AdS-CFT Correspondence, Classical Theories of Gravity, Black Holes

ARXIV EPRINT: 1401.6505

\footnotetext{
${ }^{1}$ Corresponding author.
} 


\section{Contents}

1 Introduction $\quad 1$

2 Equations of motion and boundary conditions 3

3 Insulator/superconductor phase transition 4

4 Holographic entanglement entropy $\quad 6$

4.1 Holographic entanglement entropy for a half space $\quad 7$

4.2 Holographic entanglement entropy for a strip shape 8

$\begin{array}{lll}5 & \text { Summary } & 11\end{array}$

\section{Introduction}

The anti-de Sitter/conformal field theories (AdS/CFT) correspondence [1-3] has provided us a useful theoretical method to study the strongly coupled systems in various fields of physics. In recent years, it has been widely applied to study the holographic model of superconductors [4-6]. It is indicated that the bulk AdS black hole becomes unstable and scalar hair condenses as one tunes the temperature of black hole. Besides the bulk AdS black hole spacetime, since the AdS soliton configuration has the same boundary topology as the Ricci flat black hole and the AdS space in the Poincaré coordinates, a superconductor phase dual to an AdS soliton configuration without backreaction was studied in ref. [7]. It was shown that if one increases a chemical potential $\mu$ to the AdS soliton, there is a second order phase transition at a critical value $\mu_{c}$ beyond which the charge scalar field turns on, even at zero temperature. Considering the backreaction of the matter fields on the soliton geometry, a first order phase transition occurs for the same chemical potential as the backreaction is strong enough [8]. When thinking about the higher order correction to the Maxwell field, it is of interest to investigate the effect of the Born-Infeld electrodynamics [9-14] on the insulator/superconductor transition. The Born-Infeld electrodynamics, which was proposed in 1934 to avoid the infinite self-energies for charged point particles arising in Maxwell theory [9], displays good physical properties including the absence of shock waves and birefringence. It was found that the Born-Infeld electrodynamics is the only possible non-linear version of electrodynamics that is invariant under electromagnetic duality transformation [10]. The Lagrangian density for Born-Infeld theory is $L_{\mathrm{BI}}=\frac{1}{b^{2}}\left(1-\sqrt{1+\frac{b^{2} F^{a b} F_{a b}}{2}}\right)$ with $F^{2}=F_{\mu \nu} F^{\mu \nu}$ and this Lagrangian will reduce to the Maxwell case as the coupling parameter $b$ approaches zero. In the limit of the probe approximation, the Born-Infeld factor $b$ has nothing to do with the insulator/superconductor 
transition [16]. In this paper we will extend the previous works by considering the full backreaction of the matter fields with the Born-Infeld electrodynamics on the soliton geometry.

On the other hand, the entanglement entropy has become a useful quantity for studying some properties in quantum field theories and in many-body physics recently [17-23]. As is well known, dividing a given quantum system into a subsystem $\mathcal{A}$ and its complement, the entanglement entropy of $\mathcal{A}$ is defined as the von Neumann entropy. In quantum manybody physics, the entanglement entropy allows one to distinguish new topological phases and characterize critical points. In the light of the AdS/CFT correspondence, the entanglement entropy may provide us new insights into the quantum structure of spacetime [24, 25]. Ryu and Takayanagi $[26,27]$ have provided a proposal to compute the entanglement entropy of CFTs from the minimal area surface in gravity side. This proposal provides a simple and elegant way to calculate the entanglement entropy of a strongly coupled system which has a gravity dual. Then, a lot of works have been carried out for investigating the entanglement entropy in various gravity theories [28-36]. Refs. [33-35] presented the calculations of the entanglement entropy for the AdS soliton geometry. Ref. [36] considered the case with higher derivative corrections and studied the holographic entanglement entropy in GaussBonnet gravity. Ref. [37] studied the holographic entanglement entropy for general higher derivative gravity and proposed a general formula for calculating the entanglement entropy in theories dual to higher derivative gravity. Since the entanglement entropy behaves like the thermal entropy of background black holes, it can indicate not only the appearance, but also the order of the phase transition [38-40]. The authors [41] investigated the behavior of the entanglement entropy in a simple holographic insulator/superconductor model at zero temperature, and found that the entanglement entropy as a function of chemical potential is not monotonic in the superconductor phase. Precisely, the entanglement entropy first increases and reaches its maximum at a certain chemical potential and then decreases monotonically as chemical potential increases. This non-monotonic behavior is quite different from the case of the metal/superconductor phase transition [38]. Furthermore, this non-monotonic behavior of the entanglement entropy versus the chemical potential still stands in the Stückelberg holographic insulator/superconductor model [42]. Motivated by the study of the entanglement entropy in the higher correction to gravity and in the insulator/superconductor transition, it is natural to study how the entanglement entropy will be modified as a result of the correction to the Maxwell field. Here we would like to study the entanglement entropy in the insulator/superconductor phase transition with Born-Infeld electrodynamics in the two geometry configurations which are descried by the half space and the strip one, respectively. We find that the non-monotonic behavior of the entanglement entropy versus the chemical potential is universal in this model. And the entanglement entropy increases as the Born-Infeld factor $b$ increases in the superconductor phase. Particularly, the confinement/deconfinement phase transition exists in the strip geometry.

The framework of this paper is as follows. In section 2, we will introduce the holographic superconductor models and derive the equations of motions. In section 3, we will study the phase transition in the AdS soliton gravity with Born-Infeld electrodynamics. In section 4, we will calculate the holographic entanglement entropy for the half geometry and strip space respectively. In section 5, we will conclude our main results of this paper. 


\section{Equations of motion and boundary conditions}

Let us begin with the action for a Born-Infeld electromagnetic field coupling with a charged scalar field with a negative cosmological constant in five-dimensional spacetime

$$
\begin{array}{r}
S=\int d^{5} x \sqrt{-g}\left(R+\frac{12}{L^{2}}\right)+\int d^{5} x \sqrt{-g}\left[\frac{1}{b^{2}}\left(1-\sqrt{1+\frac{b^{2} F^{a b} F_{a b}}{2}}\right)\right. \\
\left.-|\nabla \Psi-i q A \Psi|^{2}-m^{2}|\Psi|^{2}\right]
\end{array}
$$

where $L$ is the radius of AdS spacetime, $g$ is the determinant of the metric, $q$ and $m$ are respectively the charge and the mass of the scalar field, and $b$ is the Born-Infeld coupling parameter. Since we are interested in including the backreaction, we will choose the ansatz of the geometry of the AdS soliton with the form

$$
d s^{2}=\frac{d r^{2}}{r^{2} B(r)}+r^{2}\left[-e^{C(r)} d t^{2}+d x^{2}+d y^{2}+e^{A(r)} B(r) d \chi^{2}\right] .
$$

Without loss of generality, we set $L=1$ in this paper. As in the usual AdS soliton, to get a smooth geometry at the tip $r_{0}, \chi$ should be periodic with the period

$$
\Gamma=\frac{4 \pi e^{-A\left(r_{0}\right) / 2}}{r_{0}^{2} B^{\prime}\left(r_{0}\right)}
$$

and $B(r)$ vanishes at the tip of the soliton. The electromagnetic field and the scalar field can be taken as

$$
A_{t}=\phi(r), \quad \psi=\psi(r) .
$$

The equations of motion under the above ansatz can be obtained as follows

$$
\begin{aligned}
& \psi^{\prime \prime}+\left(\frac{5}{r}+\frac{A^{\prime}}{2}+\frac{B^{\prime}}{B}+\frac{C^{\prime}}{2}\right) \psi^{\prime}+\frac{1}{r^{2} B}\left(\frac{e^{-C} q^{2} \phi^{2}}{r^{2}}-m^{2}\right) \psi=0, \\
& \phi^{\prime \prime}+\left(\frac{3}{r}+\frac{A^{\prime}}{2}+\frac{B^{\prime}}{B}-\frac{C^{\prime}}{2}\right) \phi^{\prime}-b^{2} e^{-C} B\left(\frac{3}{r}+\frac{A^{\prime}}{2}+\frac{B^{\prime}}{2 B}\right) \phi^{\prime 3} \\
& -\frac{2 \psi^{2} q^{2} \phi}{r^{2} B}\left(1-b^{2} e^{-C} B \phi^{2}\right)^{\frac{3}{2}}=0, \\
& A^{\prime}=\frac{2 r^{2} C^{\prime \prime}+r^{2} C^{\prime 2}+4 r C^{\prime}+4 r^{2} \psi^{\prime 2}-2 e^{-C} \phi^{\prime 2}\left(1-b^{2} e^{-C} B \phi^{\prime 2}\right)^{-\frac{1}{2}}}{r\left(6+r C^{\prime}\right)} \text {, } \\
& C^{\prime \prime}+\frac{1}{2} C^{\prime 2}+\left(\frac{5}{r}+\frac{A^{\prime}}{2}+\frac{B^{\prime}}{B}\right) C^{\prime}-\left[\phi^{\prime 2}\left(1-b^{2} e^{-C} B \phi^{\prime 2}\right)^{-\frac{1}{2}}\right. \\
& \left.+\frac{2 q^{2} \phi^{2} \psi^{2}}{r^{2} B}\right] \frac{e^{-C}}{r^{2}}=0 \\
& B^{\prime}\left(\frac{3}{r}-\frac{C^{\prime}}{2}\right)+B\left[\psi^{\prime 2}-\frac{1}{2} A^{\prime} C^{\prime}+\frac{1-\left(1-b^{2} e^{-C} B \phi^{\prime 2}\right)^{\frac{1}{2}}}{r^{2} b^{2} B\left(1-b^{2} e^{-C} B \phi^{\prime 2}\right)^{\frac{1}{2}}}+\frac{12}{r^{2}}\right] \\
& +\frac{1}{r^{2}}\left(\frac{e^{-C} q^{2} \phi^{2} \psi^{2}}{r^{2}}+m^{2} \psi^{2}-12\right)=0
\end{aligned}
$$


where a prime denotes the derivative with respect to $r$. By considering a series solution about the tip of the soliton $\left(r=r_{0}\right)$ and using the boundary condition $B\left(r_{0}\right)=0$, we get four independent parameters, i.e., $r_{0}, \psi\left(r_{0}\right), \phi\left(r_{0}\right)$ and $C\left(r_{0}\right)$. Interestingly, we note that the above equations of motion have useful scaling symmetries

$$
\begin{array}{rlrlrl}
r & \rightarrow \alpha r, & (\chi, x, y, t) & \rightarrow(\chi, x, y, t) / \alpha, & & \phi \rightarrow \alpha \phi, \\
C & \rightarrow C-2 \ln \beta, & & \rightarrow \beta t, & \phi \rightarrow \phi / \beta .
\end{array}
$$

Therefore, we can pick any values for the position of the tip $r_{0}$ and $C\left(r_{0}\right)$. Here, we take $r_{0}=1, C\left(r_{0}\right)=0$ for simplicity.

At the spatial infinity $(r \rightarrow \infty)$, as we want the spacetime to be asymptotically AdS, the matter fields have the form

$$
\begin{aligned}
& \phi \sim \mu-\frac{\rho}{r^{2}}, \\
& \psi \sim \frac{\psi_{-}}{r^{\Delta_{-}}}+\frac{\psi_{+}}{r^{\Delta_{+}}},
\end{aligned}
$$

where the conformal dimensions of the operators are $\Delta_{ \pm}=2 \pm \sqrt{4+m^{2}}, \mu$ and $\rho$ are the corresponding chemical potential and charge density in the dual field theory, respectively. According to the AdS/CFT correspondence, the coefficients $\psi_{-}$and $\psi_{+}$correspond to the vacuum expectation values $\psi_{-}=<\mathcal{O}_{-}>, \psi_{+}=<\mathcal{O}_{+}>$of an operator $\mathcal{O}$ dual to the scalar field. For the sake of obtaining the stability in the asymptotic AdS region, we can impose boundary conditions that either $\psi_{-}$or $\psi_{+}$vanishes. In addition, in five-dimensional spacetime, when $-4<m^{2}<-3$, the scalar field admits two different quantization related by a Legendre transform [43]. Hence, we will focus on the case $m^{2}=-15 / 4, q=2$ and $\psi_{-}=0$ in the following calculation. Furthermore, to recover the pure AdS boundary, we also need $A(r \rightarrow \infty)=0$ and $C(r \rightarrow \infty)=0$.

Using the scaling symmetries (2.10), we can rescale the quantities as

$$
\Gamma \rightarrow \frac{1}{\alpha} \Gamma, \quad \mu \rightarrow \alpha \mu, \quad \rho \rightarrow \alpha^{3} \rho, \quad\left\langle\hat{O}_{2}\right\rangle \rightarrow \alpha^{\frac{5}{2}}\left\langle\hat{O}_{2}\right\rangle
$$

In the next section, we will be centered on the following dimensionless quantities $\mu \Gamma, \rho \Gamma^{3}$ and $\left\langle\hat{O}_{2}\right\rangle^{\frac{2}{5}} \Gamma$.

\section{Insulator/superconductor phase transition}

To study the phase transition for Born-Infeld electrodynamics with full backreaction in the five-dimensional AdS soliton spacetime, we make a coordinate transformation from $r$-coordinate to $z$-coordinate by defining $z=r_{0} / r$. Then, the equations of motion can be 
rewritten as

$$
\begin{gathered}
\psi^{\prime \prime}+\left(\frac{A^{\prime}}{2}+\frac{B^{\prime}}{B}+\frac{C^{\prime}}{2}-\frac{3}{z}\right) \psi^{\prime}+\frac{1}{z^{2} B}\left(e^{-C} q^{2} \phi^{2} z^{2}-m^{2}\right) \psi=0 \\
\phi^{\prime \prime}+\left(\frac{A^{\prime}}{2}+\frac{B^{\prime}}{B}-\frac{C^{\prime}}{2}-\frac{1}{z}\right) \phi^{\prime}-b^{2} z^{4} e^{-C} B\left(\frac{A^{\prime}}{2}+\frac{B^{\prime}}{2 B}-\frac{3}{z}\right) \phi^{\prime 3} \\
-\frac{2 \psi^{2} q^{2} \phi}{z^{2} B}\left(1-b^{2} z^{4} e^{-C} B \phi^{\prime 2}\right)^{\frac{3}{2}}=0 \\
\left.A^{\prime}=-\frac{z\left[2 C^{\prime \prime}+C^{\prime 2}+4 \psi^{\prime 2}-2 z^{2} e^{-C} \phi^{\prime 2}\left(1-b^{2} z^{4} e^{-C} B \phi^{\prime 2}\right)^{-\frac{1}{2}}\right]}{\left(6-z C^{\prime}\right)}+\frac{2 q^{2} \phi^{2} \psi^{2}}{B}\right] e^{-C}=0 \\
C^{\prime \prime}+\frac{C^{\prime 2}}{2}+\left(\frac{A^{\prime}}{2}+\frac{B^{\prime}}{B}-\frac{3}{z}\right) C^{\prime}-\left[z^{2} \phi^{\prime 2}\left(1-b^{2} z^{4} e^{-C} B \phi^{\prime 2}\right)^{-\frac{1}{2}}\right. \\
B^{\prime}\left(\frac{3}{z}+\frac{C^{\prime}}{2}\right)-B\left[\psi^{\prime 2}-\frac{1}{2} A^{\prime} C^{\prime}+\frac{1-\left(1-b^{2} z^{4} e^{-C} B \phi^{\prime 2}\right)^{\frac{1}{2}}}{\left.z^{2} b^{2} B\left(1-b^{2} z^{4} e^{-C} B \phi^{\prime 2}\right)^{\frac{1}{2}}+\frac{12}{z^{2}}\right]}-\frac{1}{z^{2}}\left(e^{-C} q^{2} z^{2} \phi^{2} \psi^{2}+m^{2} \psi^{2}-12\right)=0\right.
\end{gathered}
$$

where the prime now denotes a derivative with respect to $z$. The region $r_{0}<r<\infty$ now corresponds to $1>z>0$. From above discussion, by choosing $\phi\left(r_{0}\right)$ as a shooting parameter, we can solve the equations of motion for the given $m^{2}, q, \psi\left(r_{0}\right)$. After solving the equations of motion, according to the AdS/CFT correspondence, we can get the chemical potential $\mu$ and the charge density $\rho$ from the asymptotic behavior of $\phi$ through eq. (2.12). We can also get the vacuum expectation value of the scalar operator $\left.(<\mathcal{O}\rangle=\psi_{+}\right)$from eq. (2.13). For each choice of $\psi\left(r_{0}\right)$, we can solve the equations of motion for $\psi, \phi, A, B, C$. Here, we present the solutions of these equations for the factor $\psi_{0}=1.5$ by figures. In figure $1, \psi(z)$ and $\phi(z)$ are the scalar field and static electric potential, respectively, and $A(z)$ and $B(z)$ are two metric functions. For the fixed $z$, we can see that the functions have different values with the increase of the Born-Infeld factor $b$. So it is of interest to study the effect of the Born-Infeld factor $b$ on the phase transition in this system. However, the solutions obtained in this way have different periods $\Gamma$ for the $\chi$-coordinate. With the aim of comparing different solutions for the same boundary behavior, we can use the symmetry (2.14) to set all of the periods $\Gamma$ equal. As the identification length $\Gamma$ in the pure soliton is $\pi$, we will scale all $\Gamma$ for each solution to be $\Gamma=\pi$ from now on. Note that the tip $r_{0}$ will be no longer at $r_{0}=1$ after the scaling transformation. In figure 2 , we show the numerical behaviors of condensate and the charge density with the changes of the chemical potential and the Born-Infeld parameter $b$. It can be seen from the left plot of figure 2 that as the chemical potential $\mu$ exceeds a critical value $\mu_{c}$ for the given mass and charge, the condensation of the operators emerges. This can be identified as a superconductor phase. However, when less than $\mu_{c}$, the scalar field is vanishing and this can be thought of as the insulator phase. From the right plot of figure 2, we can see that the insulator/superconductor phase transition here is typically the second transition in our choice of parameters. At the critical chemical potential point, the critical value $\mu_{c}$ dose not 

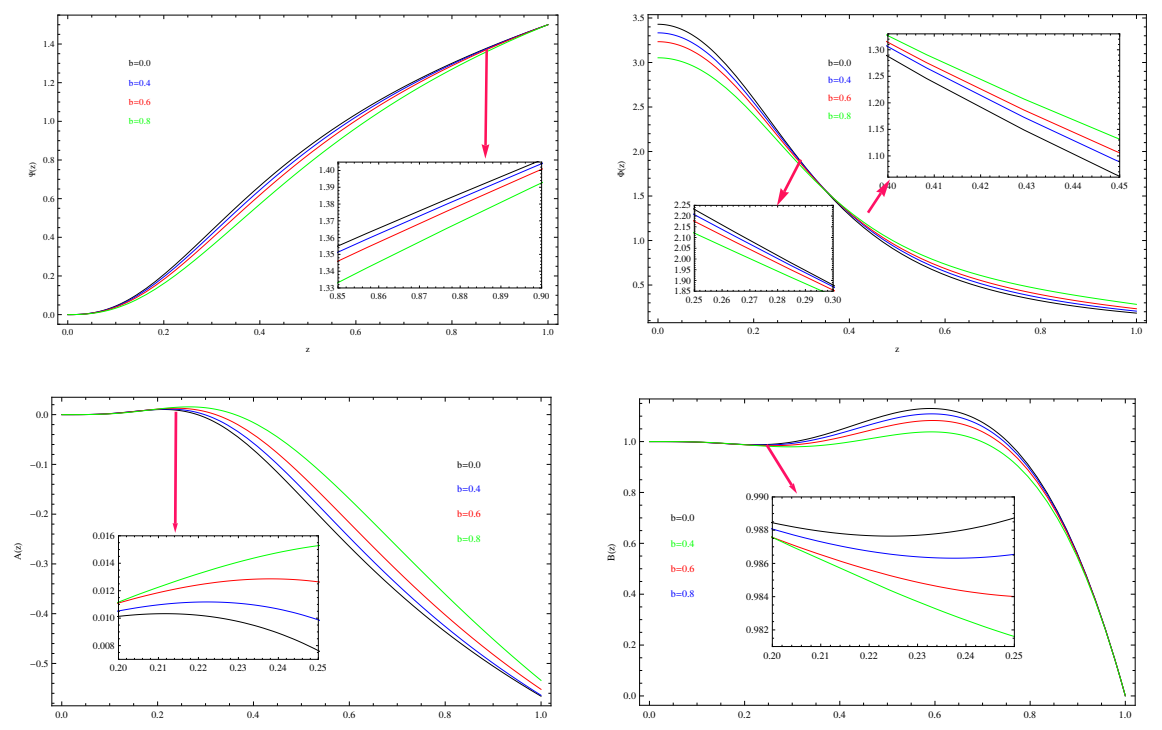

Figure 1. A typical soliton solution with nonvanishing scalar hair for the different values of parameter $b$. Here the value of the scalar field at the tip is $\psi_{0}=1.5$. The four lines from bottom to top correspond to increasing Born-Infeld factor, i.e., $\mathrm{b}=0$ (black), 0.4 (blue), 0.6 (red) and 0.8 (green), and the corresponding identifications are $\Gamma \simeq 2.4478, \Gamma \simeq 2.4455, \Gamma \simeq 2.4310$ and $\Gamma \simeq$ 2.4092 , respectively.
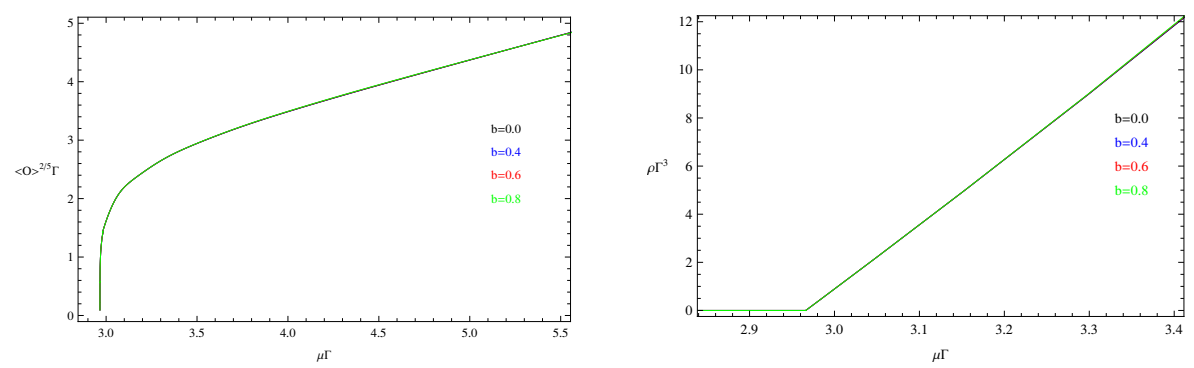

Figure 2. The condensate of operator $\left\langle\hat{O}_{+}\right\rangle$(left plot) and charge density $\rho$ (right plot) versus the chemical potential for different parameters $b$, respectively. Here we set $m^{2}=-15 / 4, q=2$ and the critical chemical potential in this case is $\mu_{c} \simeq 2.9662$. The four lines from bottom to top correspond to increasing Born-Infeld factor, i.e., $\mathrm{b}=0$ (black), 0.4 (blue), 0.6 (red) and 0.8 (green) respectively.

change with the increase of the factor $b$, which implies that the critical chemical potentials $\mu_{c}$ is independent of the Born-Infeld parameter $b$.

\section{Holographic entanglement entropy}

In this section, we study the holographic entanglement entropy in this holographic model and investigate the effect of the Born-Infeld parameter $b$ on the entanglement entropy. The holographic method to calculate the entanglement entropy is as follows. Consider a strongly coupled field theory with gravity dual, the entanglement entropy of a subsystem $\mathcal{A}$ with its complement is given by searching for the minimal area surface $\mathcal{A}$ extended into the bulk with the same boundary $\partial \mathcal{A}$ of $\mathcal{A}$. The entanglement entropy of $\mathcal{A}$ with its 
complement is given by

$$
S_{\mathcal{A}}=\frac{\operatorname{Area}\left(\gamma_{\mathcal{A}}\right)}{4 G_{N}}
$$

where $G_{N}$ is the Newton's constant in the bulk. Due to the fact that the choice of the subsystem $\mathcal{A}$ is arbitrary, we can define infinite entanglement entropies accordingly.

\subsection{Holographic entanglement entropy for a half space}

We consider a simple case where $\mathcal{A}$ is chosen to be a half of the total space. We assume that the subsystem is defined by $x>0$ and extended in the $y$ and $\chi$ directions, where $-\frac{R}{2}<y<\frac{R}{2}(R \rightarrow \infty), 0 \leq \chi \leq \Gamma$. The entanglement entropy can be deduced from the formula (4.1) as

$$
S_{\mathcal{A}}^{\text {half }}=\frac{R \Gamma}{4 G_{N}} \int_{r_{0}}^{\frac{1}{\epsilon}} r e^{\frac{A(r)}{2}} d r
$$

where $r=\frac{1}{\epsilon}$ is the UV cutoff. Note that the UV behavior of $S_{\mathcal{A}}$ will not change after the operator condensation. For the pure AdS soliton solution [41], its entanglement entropy has two parts which are the divergent part and the convergent one respectively. The divergent part of the entropy known as the area law will not change since the new solution after the operator condensation still asymptotically approaches to AdS space near the AdS boundary. However, the convergent part is the difference between the entropy in the pure AdS soliton and the one in the pure AdS space. This implies that the entropy in the AdS soliton is less than the one in the pure AdS space. Consequently, the general expression for the entanglement entropy in the half embedding case is

$$
S_{\mathcal{A}}^{\text {half }}=\frac{R \Gamma}{4 G_{N}} \int_{r_{0}}^{\frac{1}{\epsilon}} r e^{\frac{A(r)}{2}} d r=\frac{R \pi}{8 G_{N}}\left(\frac{1}{\epsilon^{2}}+s\right),
$$

where $s$ has no divergence and $s=-1$ corresponds to the pure AdS soliton. In our following numerical calculations, we set $z_{s}=\frac{r_{0}}{r}$ to require that the lower bound of the integral is still equal to unit after the scaling transformation. Then, the entanglement entropy can be rewritten as

$$
S_{\mathcal{A}}^{\text {half }}=-\frac{R \Gamma}{4 G_{N}} \int_{1}^{\epsilon r_{0}} \frac{r_{0}^{2} e^{\frac{A\left(z_{s}\right)}{2}}}{z_{s}^{3}} d z_{s}=\frac{R \pi}{8 G_{N}}\left(\frac{1}{\epsilon^{2}}+s\right) .
$$

We draw the picture of the entanglement entropy with respect to the chemical potential and the Born-Infeld factor in the dimensionless factors $s \Gamma^{2}, \mu \Gamma$ and $b$. At the critical chemical point $\mu_{c}$ which is presented by the vertical dotted green line in figure 3 , the entanglement entropy is continuous but its slope has a discontinuity. As a result, this phase transition can be regarded as the second order one. And the value of the critical chemical point $\mu_{c}$ dose not change as we increase the Born-Infeld factor $b$. This indicates that the Born-Infeld factor has no effect on the critical chemical potential. Before the phase transition, the value of the entanglement entropy $s$ presented by the dotted red line in figure 3 is a constant which indicates that this is the insulator phase. After the phase transition, when the factor $b=0$, i.e., the Born-Infeld field reduces to the Maxwell field, our results are the same as that in the ref. [41]. For the fixed $b$, with the increase of the 


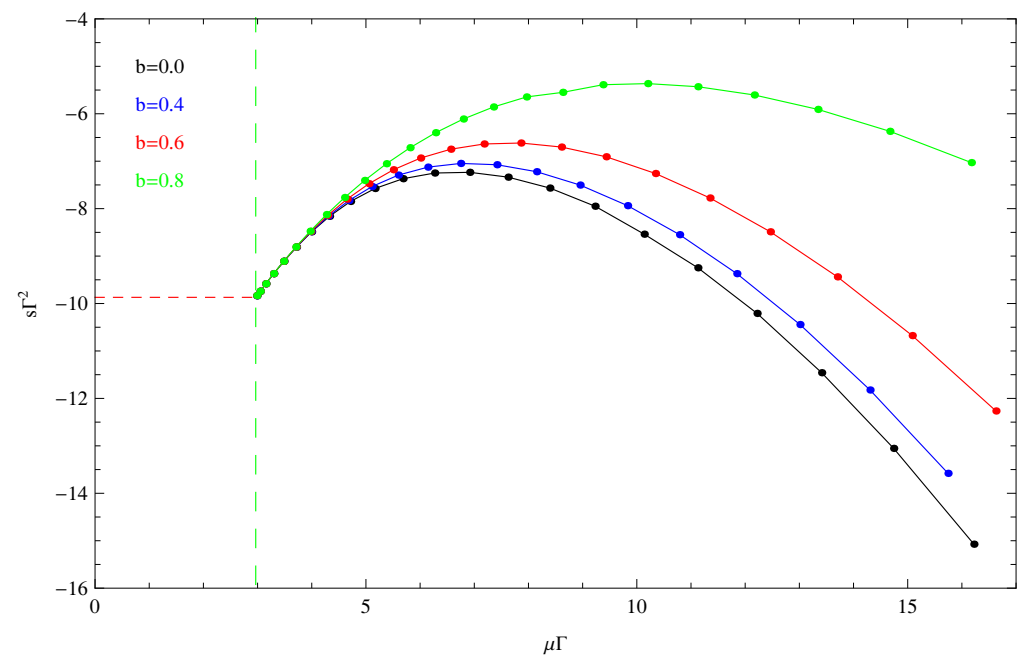

Figure 3. The entanglement entropy as a function of the chemical potential and Born-Infeld factor respectively. The four lines from bottom to top correspond to increasing Born-Infeld factor, i.e., b $=0$ (black), 0.4 (blue), 0.6 (red) and 0.8 (green) respectively.

chemical potential $\mu$, the entanglement entropy $s$ first rises and forms a peak, then decreases monotonously. This process implies that there is some kind of significant reorganization of the degrees of freedom. When the parameter $\mu$ is fixed, the entanglement entropy $s$ increases as the Born-Infeld factor $b$ increases.

\subsection{Holographic entanglement entropy for a strip shape}

After the holographic studies of entanglement entropy with the Born-Infeld electrodynamics in the half embedding, it is of interest to investigate the holographic entanglement entropy in a strip geometry. We now consider a belt shape for region $\mathcal{A}$ which is described by $-\frac{\ell}{2} \leq x \leq \frac{\ell}{2}, \quad-\frac{R}{2}<y<\frac{R}{2}(R \rightarrow \infty)$, where $\ell$ is defined as the size of region $\mathcal{A}$. The holographic dual surface $\gamma_{\mathcal{A}}$ defined as a three-dimensional surface is given by

$$
t=0, \quad x=x(r), \quad-\frac{R}{2}<y<\frac{R}{2}(R \rightarrow \infty), \quad 0 \leq \chi \leq \Gamma .
$$

The holographic surface $\gamma_{\mathcal{A}}$ starts from $x=\frac{\ell}{2}$ at $r=\frac{1}{\epsilon}$, extends into the bulk until it reaches $r=r_{*}$, then returns back to the AdS boundary $r=\frac{1}{\epsilon}$ at $x=-\frac{\ell}{2}$. Thus, the induced metric on $\gamma_{\mathcal{A}}$ can be obtained

$$
d s^{2}=h_{i j} d x^{i} d x^{j}=\left[\frac{1}{r^{2} B(r)}+r^{2}\left(\frac{d x}{d r}\right)^{2}\right] d r^{2}+r^{2} d y^{2}+r^{2} e^{A(r)} B(r) d \chi^{2} .
$$

By using the proposal (4.1) and the boundary condition (4.5), the entanglement entropy in the strip geometry is

$$
S_{\mathcal{A}}[x]=\frac{R \Gamma}{2 G_{N}} \int_{r_{*}}^{\frac{1}{\epsilon}} r e^{\frac{A(r)}{2}} \sqrt{1+r^{4} B(r)(d x / d r)^{2}} d r .
$$


Since there are several extremal surfaces, we can deduce the equation of motion for the minimal surface from eq. (4.7)

$$
\frac{r^{5} e^{\frac{A(r)}{2}} B(r)(d x / d r)}{\sqrt{1+r^{4} B(r)(d x / d r)^{2}}}=r_{s}^{3} e^{\frac{A\left(r_{s}\right)}{2}} \sqrt{B\left(r_{s}\right)},
$$

where $r_{s}$ is a constant. It is of interest to study the case that the surface is smooth at $r=r_{*}$, i.e., $d x /\left.d r\right|_{r=r_{*}}$ gets divergent. Then, the width $\ell$ of the subsystem $\mathcal{A}$ and the entanglement entropy $S_{\mathcal{A}}$ can be obtained in $z_{s}$-coordinate

$$
\begin{aligned}
\frac{\ell}{2} & =-\int_{z_{s *}}^{r_{0} \epsilon} \frac{1}{r_{0} \sqrt{B\left(z_{s}\right)\left[\frac{F\left(z_{s}\right)}{F\left(z_{s *}\right)}-1\right]}} d z_{s}, \\
S_{\mathcal{A}} & =-\frac{R \Gamma}{2 G_{N}} \int_{z_{s *}}^{r_{0} \epsilon} \frac{r_{0}^{2}}{z_{s}^{3}} e^{\frac{A\left(z_{s}\right)}{2}} \sqrt{1-\frac{F\left(z_{s *}\right)}{F\left(z_{s}\right)}} d z_{s}+\frac{R \Gamma \ell}{4 G_{N}} \sqrt{F\left(z_{s *}\right)} r_{0}^{3}=\frac{R \pi}{4 G_{N}}\left(\frac{1}{\epsilon^{2}}+s\right),
\end{aligned}
$$

where $z_{s *}=\frac{r_{0}}{r_{*}}$, and $F\left(z_{s}\right)$ is

$$
F\left(z_{s}\right)=\frac{1}{z_{s}^{6}} B\left(z_{s}\right) e^{A\left(z_{s}\right)} .
$$

There is also a disconnected solution describing two separated surfaces that are located at $x= \pm \frac{\ell}{2}$, respectively. The entanglement entropy for this disconnected geometry which is independent of $\ell$ is just twice of the half embedding solution (4.3) that we have discussed above. Moreover, the entanglement entropy in insulator/superconductor transition with Born-Infeld electrodynamics for the strip geometry is related to the chemical potential, the width of the subsystem $\mathcal{A}$ and the Born-Infeld parameter. Here, we use the diagrams to show the relationships among the dimensionless quantities $s \Gamma^{2}, \ell \Gamma^{-1}, \mu \Gamma$ and $b$. The lefthand picture of figure 4 shows the behavior of the entanglement entropy as a function of chemical potential and width for $b=0.3$. With the increase of the width $\ell$, the entanglement entropy $s$ increases but the value of $\mu_{\max }$ becomes bigger. The curve will flatten out and finally become a line in the limit $\ell \rightarrow 0$ as the length decreases. In addition, the behavior of the entanglement entropy with respect to the chemical potential is similar to the case in the half geometry. Specifically, as the chemical potential $\mu$ increases, the entanglement entropy $s$ first rises and reaches its maximum at a certain value of chemical potential denoted as $\mu_{\max }$, then it decreases monotonously.

The right-hand picture in figure 4 shows the behavior of the entanglement entropy as a function of chemical potential and Born-Infeld factor for $\ell \Gamma^{-1}=0.165$. For a given $\mu$, if the value of the chemical potential is small, the Born-Infeld factor $b$ has a relative small effect on the entanglement entropy $s$. However, if the chemical potential is large enough, the Born-Infeld factor has the obvious effect on the entanglement entropy. With the increase of the factor $b$, the entanglement entropy $s$ becomes bigger . Note that, as the chemical potential increases, the holographic entanglement entropy first rises and arrives at its maximum and then it decreases monotonously whether the Born-Infeld electrodynamics exists or not. 

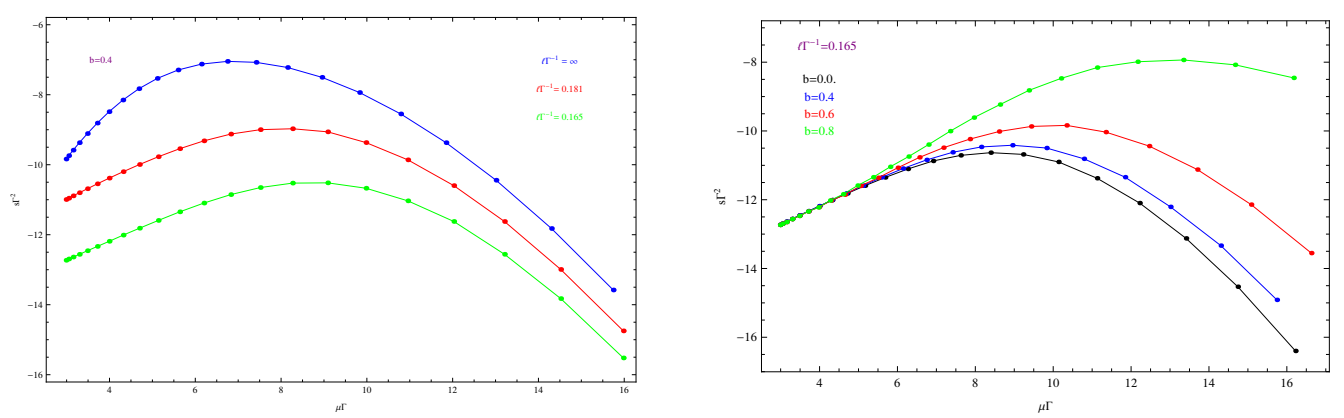

Figure 4. The entanglement entropy for the various factors, i.e., the belt width, the Born-Infeld factor and the chemical potential. For the left-hand plot, the three lines from top to bottom correspond to $\ell \Gamma^{-1}=\infty$ (blue), $\ell \Gamma^{-1}=0.181$ (red) and $\ell \Gamma^{-1}=0.165$ (green). In the right-hand one, the four lines from bottom to top correspond to $\mathrm{b}=0$ (black), 0.4 (blue), 0.6 (red) and 0.8 (green), respectively.

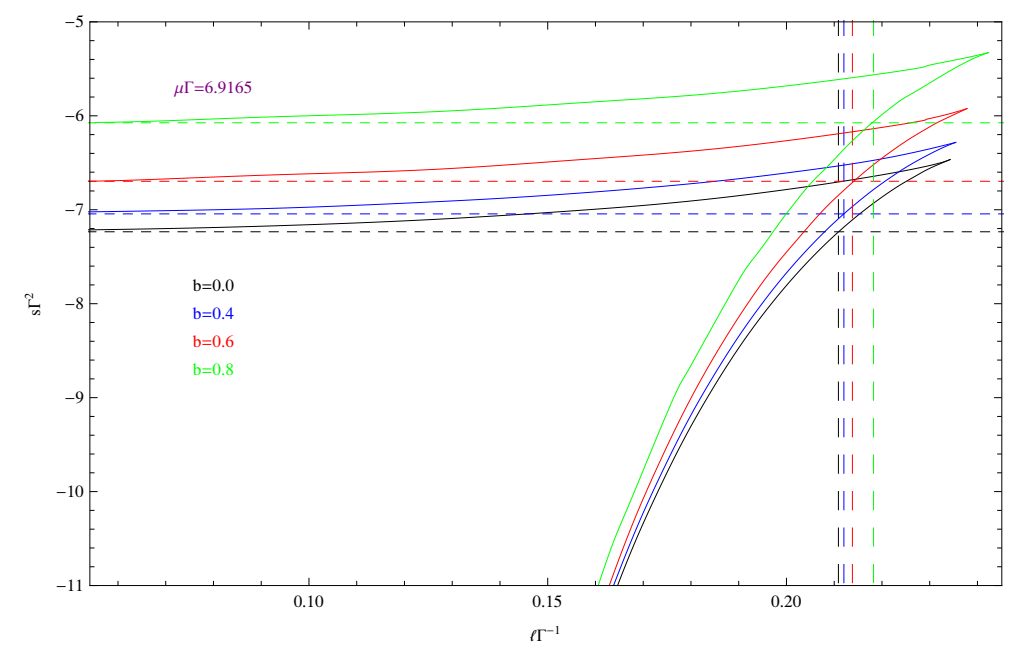

Figure 5. The entanglement entropy versus the strip width with different factors $b$ for $\mu \Gamma=6.9165$. The four lines from bottom to top correspond to increasing Born-Infeld factor, i.e., b $=0$ (black), 0.4 (blue), 0.6 (red) and 0.8 (green) respectively.

The entanglement entropy versus the strip width with different factors $b$ for $\mu \Gamma=$ 6.9165 is shown in figure 5. The horizontal dotted lines represent the discontinuous solutions, the vertical dotted lines represent the critical widths of the confinement/deconfinement phase transition and the solid one comes from the connected configuration. As the chemical potential is fixed, there exists confinement/deconfinement phase transition $[30,33,34]$ at the critical width point $\ell_{c}$. Since the physical entropy is determined by the choice of the lowest one, when $\ell<\ell_{c}$, the entanglement entropy comes from the connected surface and has the non-trivial dependence on $\ell$, which describes a deconfinement phase. And the entanglement entropy increases with the increase of the width $\ell$. As $\ell>\ell_{c}$, the entanglement entropy for disconnected configuration is favored and has nothing to do with the width $\ell$, which indicates a confinement phase. Interestingly, both in the confinement and deconfinement superconducting phase, the entanglement entropy 

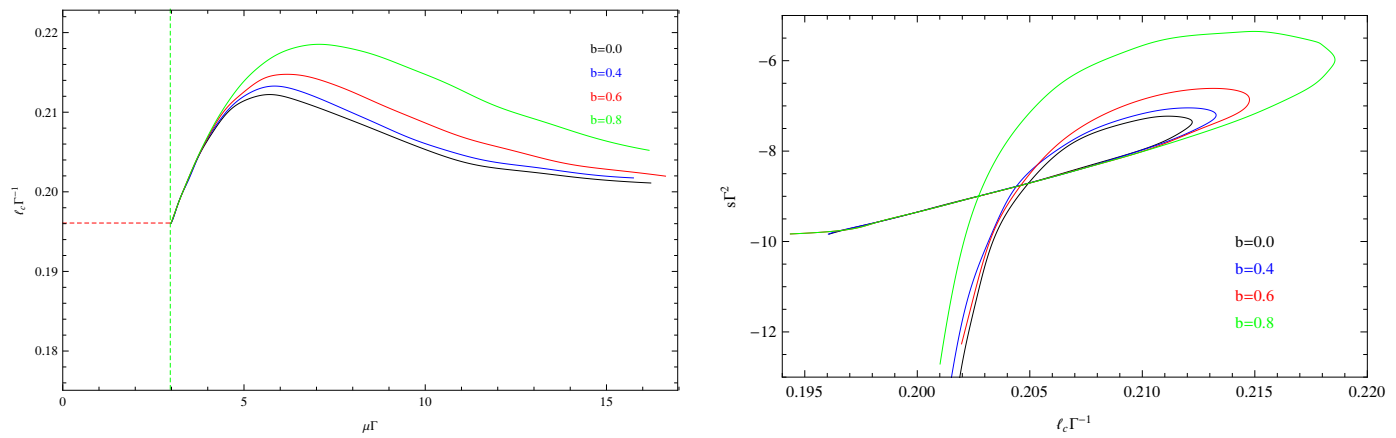

Figure 6. The left plot shows the critical belt width with respect to the chemical potential for different $b$ and the right one presents the behavior of entanglement entropy as a function of belt width at critical point of the confinement/deconfinement transition for different chemical potential. The four lines from bottom to top correspond to $b=0$ (black), 0.4 (blue), 0.6 (red) and 0.8 (green), respectively.

depends on the Born-Infeld parameter. With the increase of the factor $b$, the entanglement entropy becomes bigger. Moreover, the critical width $\ell_{c}$ increases as the factor $b$ increases for $\mu \Gamma=6.9165$.

In short, there totally exist four phases probed by the holographic entanglement entropy in the belt shape, i.e., the insulator phase, superconductor phase, and their corresponding confinement/deconfinement phases. These phases are characterized by the chemical potential and strip width. Although the Born-Infeld factor has no effect on the critical chemical potential $\mu_{c}$ of the insulator/superconductor transition, its influence on the critical width of the confinement/deconfinement phase transition is not trivial. In the insulator phase, from the left-hand plot of figure 6 , we can see that the critical length $\ell_{c}$ is a constant which means the parameter $\ell_{c}$ is independent of the Born-Infeld factor $b$. However, in the superconductor phase, the critical width $\ell_{c}$ depends both on the chemical potential $\mu$ and the Born-Infeld factor $b$. To be specific, when the chemical potential $\mu$ is fixed, the critical length $\ell_{c}$ increases with the increase of the factor $b$. For the fixed Born-Infeld factor $b$, the change of the critical width $\ell_{c}$ with the chemical potential $\mu$ is also non-monotonic but it is different from the behavior of the entanglement entropy $s$ in figure 4 . The value of the critical width $\ell_{c}$ first increases and reaches the maximum at the certain chemical potential $\mu_{\max }$, then it decreases monotonously. Note that the critical width $\ell_{c}$ decreases more and more slowly as the chemical potential $\mu$ increase. This non-monotonic behavior of the critical width versus the chemical potential is due to the fact that there is a knot which is drawn in the right-hand plot of figure 6 .

\section{Summary}

We have studied the phase transition and the holographic entanglement entropy for the Born-Infeld electrodynamics with full backreaction in five-dimensional AdS soliton spacetime. We find that the value of the critical chemical potential $\mu_{c}$ is independent of the Born-Infeld parameter $b$, which indicates that the Born-Infeld electrodynamics has no effect on the critical chemical potential of the insulator/superconductor transition. 
In the half space, at the critical point the entanglement entropy is continuous but its slope has a discontinuous change. Consequently, this phase transition can be regarded as the second order one. Before the phase transition, the value of the entanglement entropy is a constant, which indicates that it is an insulator phase. After the phase transition, for the fixed $b$, with the increase of the chemical potential $\mu$, the entanglement entropy first rises and arrives at its maximum, then decreases monotonously. This implies that there is some kind of significant reorganization of the degrees of freedom. However, when the parameter $\mu$ is fixed, the entanglement entropy increases as the Born-Infeld factor $b$ increases.

More interesting things have been found in the strip geometry. As it is shown in figure 4 , for the given $b$ or $\ell$, the non-monotonic behavior of the holographic entanglement entropy with respect to the chemical potential still holds even the Born-Infeld electrodynamics exists. While the chemical potential is fixed, the holographic entanglement entropy increases monotonously with the increase of the width of subsystem $\mathcal{A}$ and the Born-Infeld factor, respectively. From figure 5, the confinement/deconfinement phase transition exists at the critical width point $\ell_{c}$. And the value of the critical width relies on not only the critical chemical potential, but also the Born-Infeld factor. For the fixed $\mu$, the critical width increases with the increase of the Born-Infeld parameter. Interestingly, for the fixed $b$, with the increase of the chemical potential $\mu$, the critical length first increases and forms a peak, then decreases continuously. Note that, in the left-hand plot of figure 6 , the critical width $\ell_{c}$ decreases more and more slowly as the chemical potential $\mu$ increase. This non-monotonic behavior of the critical width versus the chemical potential is due to the fact that there is a knot which is drawn in the right-hand plot of figure 6 .

\section{Acknowledgments}

This work was supported by the National Natural Science Foundation of China under Grant No. 11175065; the National Basic Research of China under Grant No. 2010CB833004; the SRFDP under Grant No. 20114306110003; Hunan Provincial Innovation Foundation For Postgraduate under Grant No. CX2013A009; and Construct Program of the National Key Discipline.

Open Access. This article is distributed under the terms of the Creative Commons Attribution License (CC-BY 4.0), which permits any use, distribution and reproduction in any medium, provided the original author(s) and source are credited.

\section{References}

[1] J.M. Maldacena, The large-N limit of superconformal field theories and supergravity, Adv. Theor. Math. Phys. 2 (1998) 231 [Int. J. Theor. Phys. 38 (1999) 1113] [hep-th/9711200] [INSPIRE].

[2] S. Gubser, I.R. Klebanov and A.M. Polyakov, Gauge theory correlators from noncritical string theory, Phys. Lett. B 428 (1998) 105 [hep-th/9802109] [INSPIRE].

[3] E. Witten, Anti-de Sitter space and holography, Adv. Theor. Math. Phys. 2 (1998) 253 [hep-th/9802150] [INSPIRE]. 
[4] S.A. Hartnoll, Lectures on holographic methods for condensed matter physics, Class. Quant. Grav. 26 (2009) 224002 [arXiv:0903.3246] [INSPIRE].

[5] C.P. Herzog, Lectures on holographic superfluidity and superconductivity, J. Phys. A 42 (2009) 343001 [arXiv:0904.1975] [inSPIRE].

[6] G.T. Horowitz, Introduction to holographic superconductors, Lect. Notes Phys. 828 (2011) 313 [arXiv:1002.1722] [INSPIRE].

[7] T. Nishioka, S. Ryu and T. Takayanagi, Holographic superconductor/insulator transition at zero temperature, JHEP 03 (2010) 131 [arXiv:0911.0962] [INSPIRE].

[8] G.T. Horowitz and B. Way Complete phase diagrams for a holographic superconductor/insulator system, JHEP 11 (2010) 011 [arXiv:1007.3714] [INSPIRE].

[9] M. Born and L. Inleild, Foundations of the new field theory, Proc. R. Soc. A. 144 (1934) 425.

[10] G.W. Gibbons and D.A. Rasheed, Electric-magnetic duality rotations in non-linear electrodynamics, Nucl. Phys. 454 (1995) 185 [hep-th/9506035] [INSPIRE].

[11] B. Hoffmann, Gravitational and electromagnetic mass in the Born-Infeld electrodynamics, Phys. Rev. 47 (1935) 877 [INSPIRE].

[12] W. Heisenberg and H. Euler, Consequences of Dirac's theory of positrons, Z. Phys. 98 (1936) 714 [physics/0605038] [InSPIRE].

[13] H. de Oliveira, Nonlinear charged black holes, Class. Quant. Grav. 11 (1994) 1469 [INSPIRE].

[14] O. Miskovic and R. Olea, Conserved charges for black holes in Einstein-Gauss-Bonnet gravity coupled to nonlinear electrodynamics in AdS space, Phys. Rev. D 83 (2011) 024011 [arXiv: 1009.5763] [INSPIRE].

[15] D. Roychowdhury, Effect of external magnetic field on holographic superconductors in presence of nonlinear corrections, Phys. Rev. D 86 (2012) 106009 [arXiv:1211.0904] [INSPIRE].

[16] Z. Zhao, Q. Pan, S. Chen and J. Jing, Notes on holographic superconductor models with the nonlinear electrodynamics, Nucl. Phys. B 871 (2013) 98 [arXiv:1212.6693] [INSPIRE].

[17] P. Calabrese and J. L. Cardy, Entanglement entropy and quantum field theory, J. Stat. Mech. (2004) P06002.

[18] S. Ryu and Y. Hatsugai, Entanglement entropy and the Berry phase in the solid state, Phys. Rev. B 73 (2006) 245115 [cond-mat/0601237] [INSPIRE].

[19] M. Levin and X.-G. Wen, Detecting topological order in a ground state wave function, Phys. Rev. Lett. 96 (2006) 110405 [inSPIRE].

[20] A. Kitaev and J. Preskill, Topological entanglement entropy, Phys. Rev. Lett. 96 (2006) 110404 [hep-th/0510092] [INSPIRE].

[21] P. Calabrese and J.L. Cardy, Entanglement entropy and quantum field theory: A Non-technical introduction, Int. J. Quant. Inf. 4 (2006) 429 [quant-ph/0505193] [INSPIRE].

[22] L. Amico, R. Fazio, A. Osterloh and V. Vedral, Entanglement in many-body systems, Rev. Mod. Phys. 80 (2008) 517 [quant-ph/0703044] [INSPIRE].

[23] B. Hsu, M. Mulligan, E. Fradkin and E.-A. Kim, Universal entanglement entropy in $2 D$ conformal quantum critical points, Phys. Rev. B 79 (2009) 115421 [arXiv:0812.0203] [INSPIRE]. 
[24] M. Van Raamsdonk, Comments on quantum gravity and entanglement, arXiv:0907.2939 [INSPIRE].

[25] M. Van Raamsdonk, Building up spacetime with quantum entanglement, Gen. Rel. Grav. 42 (2010) 2323 [arXiv:1005.3035] [InSPIRE].

[26] S. Ryu and T. Takayanagi, Holographic derivation of entanglement entropy from AdS/CFT, Phys. Rev. Lett. 96 (2006) 181602 [hep-th/0603001] [INSPIRE].

[27] S. Ryu and T. Takayanagi, Aspects of holographic entanglement entropy, JHEP 08 (2006) 045 [hep-th/0605073] [INSPIRE].

[28] T. Nishioka, S. Ryu and T. Takayanagi, Holographic entanglement entropy: an overview, J. Phys. A 42 (2009) 504008 [arXiv:0905.0932] [inSPIRE].

[29] T. Albash and C.V. Johnson, Holographic entanglement entropy and renormalization group flow, JHEP 02 (2012) 095 [arXiv: 1110.1074] [INSPIRE].

[30] R.C. Myers and A. Singh, Comments on holographic entanglement entropy and RG flows, JHEP 04 (2012) 122 [arXiv: 1202.2068] [INSPIRE].

[31] J. de Boer, M. Kulaxizi and A. Parnachev, Holographic entanglement entropy in Lovelock gravities, JHEP 07 (2011) 109 [arXiv:1101.5781] [InSPIRE].

[32] L.-Y. Hung, R.C. Myers and M. Smolkin, On holographic entanglement entropy and higher curvature gravity, JHEP 04 (2011) 025 [arXiv: 1101.5813] [INSPIRE].

[33] T. Nishioka and T. Takayanagi, AdS bubbles, entropy and closed string tachyons, JHEP 01 (2007) 090 [hep-th/0611035] [INSPIRE].

[34] I.R. Klebanov, D. Kutasov and A. Murugan, Entanglement as a probe of confinement, Nucl. Phys. B 796 (2008) 274 [arXiv:0709.2140] [InSPIRE].

[35] A. Pakman and A. Parnachev, Topological entanglement entropy and holography, JHEP 07 (2008) 097 [arXiv: 0805.1891] [INSPIRE].

[36] N. Ogawa and T. Takayanagi, Higher derivative corrections to holographic entanglement entropy for AdS solitons, JHEP 10 (2011) 147 [arXiv: 1107.4363] [INSPIRE].

[37] X. Dong, Holographic entanglement entropy for general higher derivative gravity, JHEP 01 (2014) 044 [arXiv:1310.5713] [INSPIRE].

[38] T. Albash and C.V. Johnson, Holographic studies of entanglement entropy in superconductors, JHEP 05 (2012) 079 [arXiv: 1202.2605] [INSPIRE].

[39] R.-G. Cai, S. He, L. Li and Y.-L. Zhang, Holographic entanglement entropy on p-wave superconductor phase transition, JHEP 07 (2012) 027 [arXiv: 1204.5962] [INSPIRE].

[40] L.-F. Li, R.-G. Cai, L. Li and C. Shen, Entanglement entropy in a holographic p-wave superconductor model, arXiv:1310.6239 [INSPIRE].

[41] R.-G. Cai, S. He, L. Li and Y.-L. Zhang, Holographic entanglement entropy in insulator/superconductor transition, JHEP 07 (2012) 088 [arXiv:1203.6620] [INSPIRE].

[42] R.-G. Cai, S. He, L. Li and L.-F. Li, Entanglement entropy and Wilson loop in Stückelberg holographic insulator/superconductor model, JHEP 10 (2012) 107 [arXiv:1209.1019] [INSPIRE].

[43] I.R. Klebanov and E. Witten, AdS/CFT correspondence and symmetry breaking, Nucl. Phys. B 556 (1999) 89 [hep-th/9905104] [INSPIRE]. 